\title{
Experiencia del día mundial del riñón 2013 en el Hospital Militar Central de Bogotá: Síndrome metabólico y enfermedad renal
}

Jorge Echeverri ${ }^{1,2}$, Manuel Huérfano ${ }^{2}$, Vanesa López ${ }^{2}$, Andrés Calderón ${ }^{1}$, JG Vargas ${ }^{1,2}$, Alejandro Camargo ${ }^{1}$, Jaime Echenique ${ }^{1}$, Gustavo Quiroz ${ }^{1}$, Paula Rodríguez ${ }^{1}$, Jorge Pulido ${ }^{1}$, Paquita Méndez ${ }^{2}$, Amanda Castro ${ }^{2}$, Myriam Castañeda ${ }^{2}$, Nasly Ruiz ${ }^{2}$, Luz Mosquera ${ }^{2}$, Liseth Sánchez ${ }^{2}$, Jorge Cárdenas ${ }^{2}$, Roberto Dachiardi ${ }^{2}$

\footnotetext{
${ }^{1}$ Universidad Militar Nueva Granada, Bogotá, Colombia
${ }^{2}$ Servicio de nefrología RTS - Hospital Militar Central, Bogotá, Colombia
}

\begin{abstract}
Resumen
En la última década se han sumado voluntades en la lucha contra el avance de la enfermedad renal crónica. Con este fin se creó el Día Internacional del Riñón, para el cual se estableció como fecha conmemorativa el segundo jueves de marzo, de cada año, a partir del 2006. En el Hospital Militar Central de Bogotá, en el marco del Día Internacional del Riñón, se realizó una jornada educativa y de tamizaje de factores de riesgo renal, en población usuaria del sistema de salud de las Fuerzas Militares de Colombia. Se encontró en el 4,5\% de la población disminución de la tasa de filtración glomerular esperada para la edad, con $11 \%$ de presencia de microalbuminuria. El síndrome metabólico es una entidad frecuente en nuestro medio y en la población estudiada cerca de la mitad (52\%) de los asistentes cumplía con los criterios para establecer el diagnóstico. Se identificó asociación entre el aumento del índice de masa corporal o la elevación de la hemoglobina glicosilada y el desarrollo de microalbuminuria. Es necesario realizar campañas para el control del síndrome metabólico, con miras a controlar el riesgo subyacente para enfermedad renal crónica. Se describen los hallazgos encontrados.
\end{abstract}

Palabras clave: Enfermedad renal crónica, síndrome metabólico, microalbuminuria.

\section{Experience of World Kidney Day 2013 at the Central Military Hospital in Bogotá: Metabolic syndrome and renal disease}

\begin{abstract}
In the last decade wills have joined in the fight against the progression of chronic kidney disease. Thus, in 2006 the International Kidney Day was established, being the second Thursday of March of each year the commemorative date. In the Central Military Hospital in Bogotá, under the International Kidney Day, an educational and screening journey of renal risk factors was performed. Attendees were users of the health system of the Armed Forces of Colombia. It was found in $4.5 \%$ of the population a decline in the glomerular filtration rate according to age, with $11 \%$ presence of microalbuminuria. The metabolic syndrome is frequent in our environment and in our study population about half $(52 \%)$ of the attendees met the diagnostic criteria.
\end{abstract}


Association between increased BMI or elevated glycosylated hemoglobin and development of microalbuminuria was identified. Campaigns are needed to control the metabolic syndrome in order to control the underlying risk for chronic kidney disease.

Key words: chronic kidney disease, metabolic syndrome, microalbuminuria.

\section{Introducción}

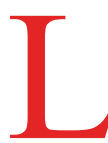

a enfermedad renal crónica (ERC) es un problema de salud pública que se ha incrementado de manera dramática través del tiempo. Según el National Health and Nutrition Examination Surveys (NHANES), la prevalencia de la ERC en Estados Unidos se incrementó de $11 \%$ en los 90 a $13 \%$ a comienzos de la década pasada, la incidencia también aumentó en países en vía de desarrollo como Colombia, lo cual se atribuye al incremento de la frecuencia de los factores de riesgo para el deterioro de la función renal, como es el caso de la prediabetes, diabetes mellitus tipo 2, hipertensión arterial y obesidad. Con este trabajo pretendemos describir la tasa de filtración glomerular estimada (TFGe), marcadores de daño renal crónico como la microalbuminuria (MA) y las características clínicas de un grupo poblacional evaluado con motivo de la celebración del Día Internacional del Riñón.

Ciento cincuenta y siete países han celebrado de forma ininterrumpida este día, bajo la iniciativa conjunta de la Sociedad Internacional de Nefrología y de la Federación Internacional de Fundaciones del Riñón, para promover la salud renal a partir de la prevención y promoción de estilos de vida saludable. El objetivo principal de esta celebración es promover la detección y el tratamiento temprano de las enfermedades renal y cardiovascular, para reducir dramáticamente otras complicaciones asociadas y los costos crecientes de muerte e invalidez.

Cada año se elige un tema para el Día Internacional del Riñón, sobre el que se desarrollan todas las iniciativas y actividades, el lema para el 2013 fue "Riñones para la vida: paremos la enfermedad renal aguda", lo cual motivó a que en nuestro país se realicen esfuerzos encaminados a continuar con este proyecto año tras año y es a partir de esto que describiremos nuestra experiencia en este documento. Para el año 2014 la campaña se denominó "Enfermedad renal crónica y envejecimiento" en la que se proyectó incluir un mayor número de personas $\mathrm{y}$, a su vez, mantenerlo como tendencia año tras año. Los resultados de esta evaluación pueden proporcionar información importante para el desarrollo de futuros programas preventivos a escala nacional, para optimizar el proceso de asignación de recursos y mejorar, además, la conciencia pública por las enfermedades relacionadas con la ERC.

\section{Objetivos}

El objetivo fue describir las características clínicas de los pacientes sometidos a exámenes de tamizaje para ERC, que asistieron de manera voluntaria a las actividades conmemorativas e informativas del Día Mundial del Riñón, celebrado el jueves 14 de marzo de 2013, en el Hospital Militar Central (HMC) de Bogotá, Colombia.

\section{Métodos}

En el Día Mundial del Riñón de 2013, el HMC realizó una jornada conmemorativa, en la que se realizaron actividades de educación, promoción de estilos de vida saludable y prevención de la enfermedad renal, a cargo de un grupo multidisciplinario conformado por profesionales en las áreas de nefrología, cardiología, endocrinología, psicología, nutrición, enfermería y trabajo social, del Servicio de Nefrología del HMC de Bogotá. Como apoyo adicional se proyectó un video elaborado y difundido por la International Society of Nephrology (ISN) y la International Federation of Kidney Foundations (IFKF) para la ocasión, el cual está disponible en www. 
worldkidneyday.org. Una vez finalizada la sesión educativa e informativa, se realizó un foro de discusión y resolución de dudas.

Los asistentes a la jornada educativa firmaron el consentimiento informado para participar en el tamizaje de ERC. Bajo el apoyo de médicos especialistas en medicina interna y personal de enfermería se realizó una encuesta de factores de riesgo para ERC, toma de signos vitales, evaluación antropométrica y toma de paraclínicos de tamizaje: pruebas hematológicas (hemograma), pruebas de función renal (nitrógeno ureico, creatinina sérica, uroanálisis y microalbuminuria) y metabólicas (porcentaje de hemoglobina glicosilada). Los pacientes, al final del día, participaron en una actividad grupal para resolución de dudas, y una vez obtenidos los resultados de la jornada se realizó la socialización de éstos a través de un comunicado institucional emitido por el jefe del servicio de nefrología, con las recomendaciones y orientaciones pertinentes. Los casos con compromiso patológico, identificados durante la jornada, fueron valorados posteriormente por el grupo de especialistas pertinente.

\section{Análisis estadístico}

En primera instancia se hizo un análisis descriptivo de la población, representando las variables numéricas con promedios y desviación estándar, y las variables categóricas como el número de personas y el porcentaje correspondientes (Tabla 1). Para evaluar la diferencia entre las medias de las variables relevantes y estratificadas se usó la prueba de $\mathrm{T}$ de Student.

\section{Resultados}

Se evaluaron un total de 224 personas, de las cuales el 55\% fueron hombres, con edad promedio de 49 años y tasa de filtración estimada por MDRD4 en promedio de 90 (Tabla 1, Figura 1). La prevalencia de antecedentes y factores de riesgo conocidos para presentar deterioro de la función renal se presenta en la tabla 2. Encontramos que $52 \%$ de los pacientes presentaba sobrepeso (IMC mayor de 25), el 15\% obesidad (IMC mayor de 30) y el 33\% tenía un IMC

\begin{tabular}{|l|c|c|}
\hline \multicolumn{2}{|c|}{ Tabla 1 } \\
\hline \multicolumn{2}{|c|}{ Características generales de la población estudiada } \\
\hline Total pacientes N & 224 & \\
\hline Mujeres N & 101 & $45 \%$ \\
\hline Hombres N & 123 & $55 \%$ \\
\hline Edad promedio (SD) & 49,3 & 16,1 \\
\hline TFG x MDRD ml/min prom (SD) & 90 & 19,4 \\
\hline IMC promedio (SD) & 27,1 & 3,6 \\
\hline HgbAlc promedio (SD) & 5,84 & 0,99 \\
\hline
\end{tabular}

Figura 1

Distribución de la TFGe por MDRD en la población estudiada

Tabla 2

\begin{tabular}{|c|c|c|}
\hline \multicolumn{3}{|c|}{$\begin{array}{l}\text { Antecedentes y factores de riesgo para desarrollo de } \\
\qquad \text { ERC }\end{array}$} \\
\hline Antecedentes y factores de riesgo renal & & \\
\hline Diabetes mellitus T2 $(\mathrm{N})$ & 17 & $7,6 \%$ \\
\hline Hipertensión arterial $(\mathrm{N})$ & 52 & $23 \%$ \\
\hline Infección de vías urinarias $(\mathrm{N})$ & 30 & $13 \%$ \\
\hline Insuficiencia cardiaca $(\mathrm{N})$ & 13 & $6 \%$ \\
\hline Enfermedad coronaria y vascular $(\mathrm{N})$ & 26 & $12 \%$ \\
\hline Uso de antiinflamatorios no esteroideos $(\mathrm{N})$ & 55 & $25 \%$ \\
\hline Uso de medio contraste $(\mathrm{N})$ & 68 & $30 \%$ \\
\hline
\end{tabular}


menor o igual a 25 (Figura 2), esto último se correlaciona también con una importante prevalencia de obesidad abdominal en 52\% de los casos (definida para la población latinoamericana como circunferencia abdominal mayor de $94 \mathrm{~cm}$ en hombres y mayor de $90 \mathrm{~cm}$ en mujeres) ${ }^{2}$ (Figura 3). La distribución de los pacientes no diabéticos según la HgbA1c se muestra en la figura 4.

En la evaluación de muestra aislada de orina, en el $89 \%$ de los pacientes no se evidenció MA, el 10\% presentó MA entre 30 y $300 \mathrm{mcg} / \mathrm{g}$ y solo el $1 \%$ tuvo MA mayor de $300 \mathrm{mcg} / \mathrm{g}$. Se evaluaron, en esta población, las características operativas del parcial de orina para detectar MA en comparación con la MA en muestra aislada, encontrándose una sensibilidad del $80 \%$, especificidad del $94 \%$, con un valor predictivo positivo del $63 \%$ y un valor predictivo negativo del 97\%.

Finalmente, se evaluó la diferencia entre las medias de microalbuminuria entre variables relevantes, encontrándose diferencias significativas entre los pacientes que tenían porcentajes de HbA1C mayor y menor de 5,5 ( $<<0,009)$, e índices de masa corporal mayores y menores de $25(\mathrm{p}<0,005)$, sin encontrarse diferencias significativas, tanto en hombres como en mujeres con obesidad abdominal (Tabla 3 ).

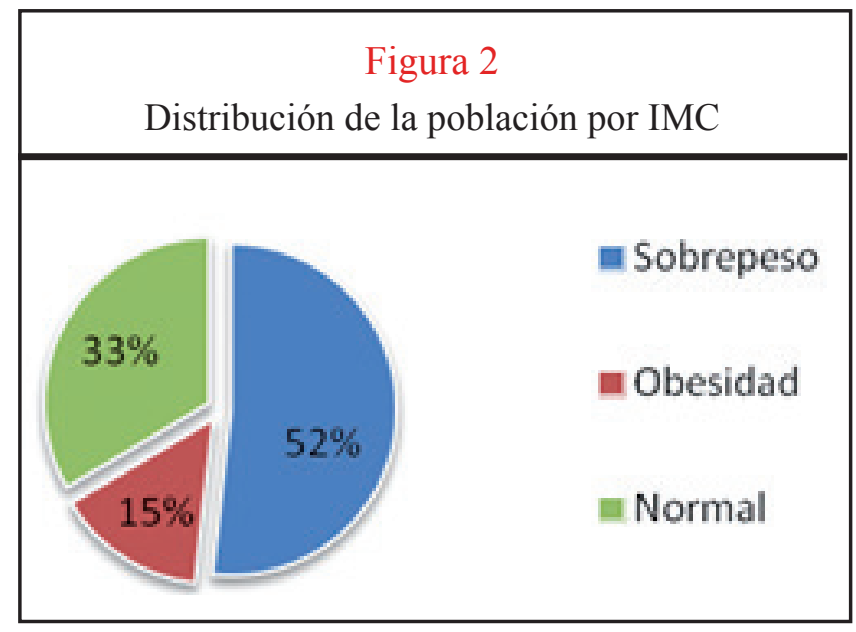

Figura 3

Distribución de la población según la presencia de obesidad abdominal

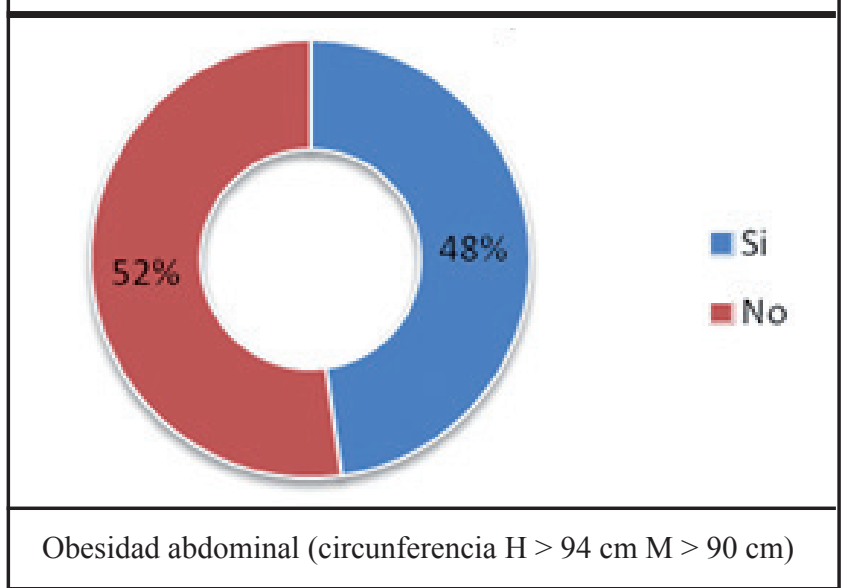

Figura 4

Distribución de los pacientes no diabéticos según la Hgba1c

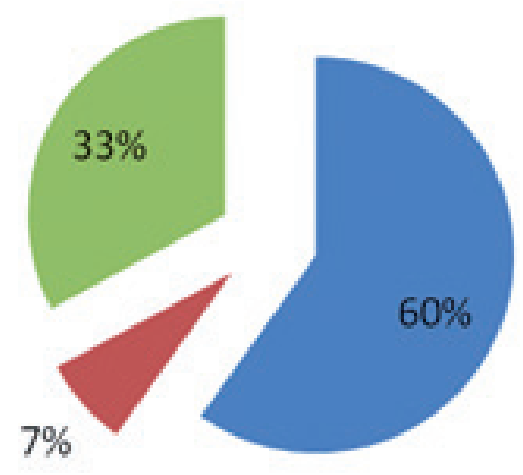

Grupo A (Sin

antecedente y $\mathrm{Hgba} 1 \mathrm{c}$

$5,5-6,4)$

Grupo B (Sin

antecenete y $\mathrm{Hgba} 1 \mathrm{c}>$

$6,5)$

Grupo C (Sin

antecedente $\mathrm{Hgba} 1 \mathrm{c}$

menos de 5,5) 


\section{Tabla 3}

\begin{tabular}{|l|c|c|c|}
\hline \multicolumn{3}{|c|}{ Diferencia entre las medias de la albuminuria entre variables relevantes } \\
\hline Variable & \multicolumn{2}{|c|}{ Albuminuria (mcg/gr) } & p \\
\hline Hgbalc $<5,5$ vs. $>$ de 5,5 & 8,00 & 21,57 & 0,0098 \\
\hline IMC $<25$ vs. $>25$ & 7,88 & 23,08 & 0,0057 \\
\hline Mujeres cintura abdominal $>90$ cm vs. $<90 \mathrm{~cm}$ & 13,91 & 12,04 & 0,6808 \\
\hline Hombres cintura abdominal $>94 \mathrm{~cm}$ vs. $<94 \mathrm{~cm}$ & 29,99 & 13,74 & 0,1904 \\
\hline
\end{tabular}

\section{Discusión}

Una de las limitantes más importantes en la prevención de la ERC, en la actualidad, es la poca conciencia o el escaso conocimiento del paciente de su enfermedad. En el programa estadounidense de la National Kidney Foundation, "Evaluación Renal Temprana" (KEEP, por sus siglas en inglés), se realizó un tamizaje de enfermedad renal a 26.213 individuos, durante 10 años de seguimiento. De este grupo, solo el $9.5 \%$ de los participantes fue consciente de tener algún grado de compromiso renal ${ }^{3}$.

A pesar de ser una pequeña muestra poblacional, encontramos que los participantes tenían una distribución de la TGFe por MDRD4 similar a las descritas en cohortes nacionales (Figura 1), con alta similitud de los principales factores de riesgo para deterioro de la función renal. Sin embargo, cuando analizamos la prevalencia de sobrepeso y obesidad, encontramos mayor proporción de individuos con estas dos condiciones (67\% sobrepeso u obesidad), junto con una alta frecuencia de obesidad abdominal (52\%). Adicionalmente, evidenciamos que $7 \%$ eran diabéticos conocidos, pero $60 \%$ presentaban $\mathrm{HgA} 1 \mathrm{C}$ en rango de prediabetes y un 7\% adicional se confirmó como diabético de novo en controles posteriores. Es así como podemos afirmar, con base en éstos resultados, que al menos el $60 \%$ de los pacientes estudiados, en nuestra población, podría cumplir criterios para síndrome metabólico 4 . Sumado a esto, el $40 \%$ de la población diabética se encontraba fuera del rango terapéutico de HgA1C esperado.

Teniendo en cuenta el incremento a escala mundial de la ERC asociada con síndrome metabólico, es necesario profundizar en esta temática. Se ha encon- trado ERC, como hallazgo incidental en diferentes estudios poblacionales. Un grupo norteamericano encontró en 6.217 participantes aumento de la proporción de deterioro de la TFGe (OR 2.60; IC95\% $1.68-4.03$ ) y presencia de MA(OR 1.89; IC95\% 1.342.67), cuando comparó los individuos con síndrome metabólico versus los que no lo tenían ${ }^{5}$. Un estudio coreano recientemente publicado, encontró desenlaces similares con incremento en el riesgo de ERC en pacientes con síndrome metabólico, tanto hombres (OR 1.45; IC95\% 1.2-1.6) como mujeres (OR 1.76; IC95\% 1.2-1.76), al compararlos con la población control. Sumado a esto, el grupo coreano mostró que dicha asociación aumentaba ante la presencia de hipertensión arterial o niveles bajos de $\mathrm{HDL}^{6}$.

Nuestra cohorte evidenció la asociación entre la presencia de aumento del índice de masa corporal $(>25)$ o elevación de la $\mathrm{HgA} 1 \mathrm{C}(>5,5)$ con el desarrollo de albuminuria. Desde el punto de vista fisiopatológico, algunos estudios afirman que, el desarrollo de ERC en las poblaciones con estas características, es un factor de riesgo independiente de otras comorbilidades como hipertensión arterial y diabetes, contrario a lo que se podría esperar. Se han descrito cambios histopatológicos en el tejido renal de pacientes con síndrome metabólico, tales como: atrofia tubular, fibrosis intersticial y esclerosis.

Varios estudios también han demostrado diferentes vías que asocian la resistencia a la insulina o hiperinsulimenia con ERC $^{6}$. Esto, se desarrolla a través de varias fases: primero, la resistencia a la insulina con hiperinsulinemia compensatoria que promueve la activación inadecuada del sistema renina-angiotensina-aldosterona, lo que induce hiperaldosteronismo 
e hipertensión glomerular. Segundo, la resistencia a la insulina, incrementa el estrés oxidativo que también se ha implicado en la progresión de la glicoxidación y la peroxidación lipídica y la resistencia a la insulina induce la proliferación mensangial y de matriz extracelular a través de la estimulación de endotelina $1 \mathrm{y}$ otros factores de crecimiento, y finalmente el hiperinsulinismo, desregula la acción renal de los receptores de peroxisomas y, con ello, se induce la formación de células espumosas, lipotoxicidad renal y disfunción endotelial.

En otras experiencias de campañas del Día Mundial del Riñón ${ }^{7}$ se resalta la importancia de un diagnóstico temprano de compromiso renal y la identificación de factores de riesgo con su manejo adecuado, para prevenir la progresión de la ERC. Es de resaltar al síndrome metabólico como un factor poco explorado en nuestra población, que debiera de servir de tamizaje inicial con pruebas sencillas encaminadas a la detección temprana del compromiso renal. Los resultados presentados nos sugieren que es necesario realizar intervenciones dirigidas a cambios en el estilo de vida y encaminadas a disminuir la prevalencia del síndrome metabólico, con el fin de controlar un posible factor precipitante de compromiso renal en nuestra población.

\section{Conclusiones}

Presentamos los resultados de la experiencia del Hospital Militar Central de Bogotá, en población usuaria del sistema de salud de las fuerzas militares, con la cual se realizaron actividades de promoción y prevención de ERC, dentro del marco del Día Mundial del Riñón.

Encontramos en 4,5\% de la población disminución de la TFGe esperada para la edad, con presencia de MA en 11\%. El uronálisis nos dio un valor predictivo positivo de $65 \%$, mientras que su valor predictivo negativo fue de $97 \%$ para detectar MA. Los factores de riesgo más relevantes para el desarrollo de ERC identificados como antecedentes en la población analizada fueron hipertensión arterial, diabetes mellitus e infección de vías urinarias, uso de antiinflamatorios no esteroideos y antecedentes de exposición a medio de contraste.

El síndrome metabólico es una entidad frecuente en nuestro medio y en la población estudiada cerca de la mitad completaba criterios para su diagnóstico. Se identificó asociación entre el aumento del índice de masa corporal o la elevación de la HgA1C y el desarrollo de microalbuminuria. Es necesario realizar campañas para el control del síndrome metabólico con miras a controlar el riesgo subyacente para ERC.

\section{Conflicto de interés}

Los autores declaran no tener ningún conflicto de interés.

\section{Referencias bibliográficas}

1. Coresh J, Selvin E, Stevens LA et al. Prevalence of chronic kidney disease in the United States. JAMA 2007;298:2038-2047.

2. Aschner P, Buendía R, Brajkovich I, González A, Figueredo R, Juárez XE, Uriza F, Gómez AM, Ponte CI. Determination of the cutoff point for waist circumference that establishes the presence of abdominal obesity in Latin American men and women. Diabetes Res Clin Pract. 2011 Aug;93(2):243-7.

3. Kurella Tamura M, Anand S, Li S, Chen SC, Whaley-Connell AT, et al. Comparison of CKD awareness in a screening population using the Modification of Diet in Renal Disease (MDRD) study and CKD Epidemiology Collaboration (CKD-EPI) equations. Am J Kidney Dis 2011;57(3S2):S17-23. 
4. Rosas Guzmán J., González Chávez A., Aschner P., Bastarrachea R. y col, Epidemiología, Diagnóstico, Control, Prevención y Tratamiento del Síndrome Metabólico en Adultos, Consenso ALAT, VOL. XVIII - N 1 - Año 2010.

5. Chen J, Muntner P, Hamm LL, et al. The metabolic syndrome and chronic kidney disease in U.S. adults. AnnIntern Med 2004;140:167-74.

6. Cho JA, Lee SJ, Reid EA, Jee SH. Metabolic syndrome component combinations and chronic kidney disease: the severance cohort study. Maturitas. 2013; 75(1):74-80.

7. Chin HJ et al, The effect of the World Kidney Day campaign on the awareness of chronic kidney disease and the status of risk factors for cardiovascular disease and renal progression, Nephrol Dial Transplant 2010;25:413-419. 